\title{
PMIP contributions to understanding the deep ocean circulation of the Last Glacial Maximum
}

\author{
Sam Sherriff-Tadano ${ }^{1}$ and Marlene Klockmann²

\begin{abstract}
Simulations of the Last Glacial Maximum (LGM) within PMIP significantly improved our understanding of the mechanisms that control the Atlantic Meridional Overturning Circulation (AMOC) in a glacial climate. Nonetheless, reproducing the reconstructed shallowing of the LGM AMOC remains a challenge for many models.
\end{abstract}

\section{AMOC at the LGM}

The Last Glacial Maximum (LGM; ca. 21,000 years ago) was a period within the last glacial cycle with very low greenhouse gas concentrations and maximum ice volume. The global climate was much colder than the modern climate, and the state of the Atlantic Meridional Overturning Circulation (AMOC) was very different as a consequence of the glacial climate forcings. In the modern climate, North Atlantic Deep Water (NADW), which forms in the Nordic and Labrador Seas, fills the deep North Atlantic basin. In contrast, proxy data such as carbon and neodymium isotopes, suggest that during the LGM, a large fraction of NADW in the deep Atlantic basin was replaced by Antarctic Bottom Water (AABW), which is formed in the Southern Ocean. As a result, the glacial AMOC was shallower than the modern AMOC (Lynch-Stieglitz 2017). The strength of the LGM AMOC is harder to reconstruct; proxies of $A M O C$ strength support a glacial $A M O C$ state ranging from weaker than or similar to today (e.g. Lynch-Stieglitz 2017). Nonetheless, the LGM provides a good opportunity to understand the AMOC response to climate changes as well as to evaluate the capability of comprehensive atmosphere-ocean coupled general circulation models (AOGCM) to reproduce AMOC states which are very different from today.

\section{LGM AMOC from PMIP1 to PMIP4}

Throughout the four PMIP phases, simulating the LGM AMOC has remained a challenge. While the respective $A O G C M$ s tend to agree on large-scale changes in surface cooling patterns, the simulated AMOC changes differ strongly between models and PMIP phases, and most models cannot simulate the reconstructed shallower LGM AMOC. The first official LGM AMOC model intercomparison was conducted as part of PMIP2 (Weber et al. 2007); this intercomparison included three additional simulations from AOGCMs that adopted the PMIP1 protocol. These simulations are referred to as PMIP1.5 simulations. Here, we include a fourth PMIP1.5-type simulation (Kim 2004) that was not part of the original intercomparison.

In Figure 1, the results of various PMIP phases are shown. Out of nine PMIP1.5/ PMIP2 models, four simulated a shallower and weaker LGM AMOC, three a stronger and deeper LGM AMOC, one simulated a stronger LGM AMOC with no changes in depth, and one a deeper and slightly weaker LGM AMOC. In PMIP3, the inter-model spread was much smaller, but fewer models agreed with reconstructions. Only one model simulated a shallower LGM AMOC, one simulated no change in depth, and all other models simulated a much deeper LGM AMOC. All models simulated a stronger LGM AMOC. In PMIP4, most models simulated a stronger LGM AMOC, while all but two models simulated very minor changes in the depth (Kageyama et al. 2021).

\section{What have we learned from PMIP?}

The PMIP ensembles have provided many plausible hypotheses regarding the mechanisms that control the LGM AMOC. While there are still open questions, it is possible to assemble some pieces of the puzzle to form a consistent picture. The PMIP1.5/ PMIP2 simulations suggested that the meridional density contrast between NADW and $A A B W$ source regions plays a key role in controlling the AMOC state (Weber et al. 2007): the glacial AABW needs to become much denser than the NADW in order to generate strong enough stratification in the deep ocean, thereby inducing a shallower AMOC. Starting from there, key processes

(A)

LGM-PI

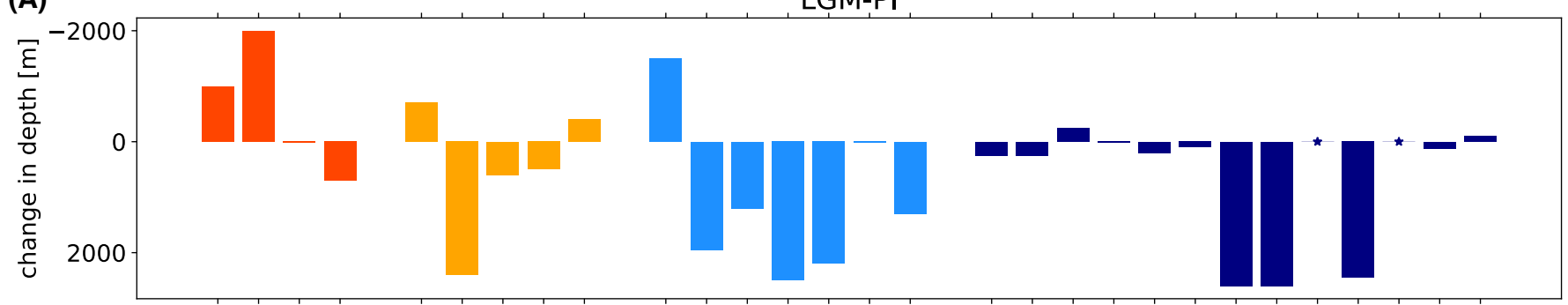

(B)

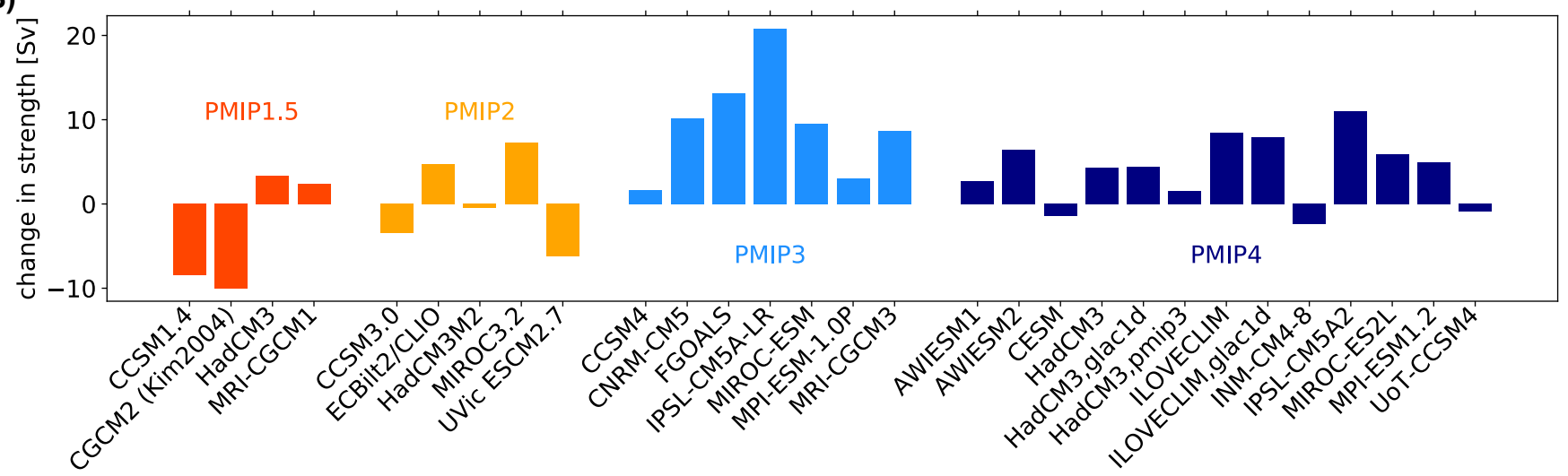

Figure 1: (A) AMOC depth and (B) strength at the LGM compared to pre-industrial (PI) in the PMIP generations 1.5-4. The values for PMIP1.5 and PMIP2 are taken from Weber et al (2007) and Kim (2004); values for PMIP3 and PMIP4 are taken from Kageyama et al (2021). AMOC strength is defined as the maximum transport in Sv at $30^{\circ} \mathrm{N}$. AMOC depth is defined as the depth of the interface between the NADW and AABW cell at $30^{\circ} \mathrm{N}$ for CGCM2, PMIP3, and PMIP4, and at the Southern end of the Atlantic basin for PMIP1.5 and PMIP2. Negative values in AMOC depth and strength correspond to shoaling and weakening of LGM AMOC compared to PI. Asterisks indicate that the NADW cell covers the entire water column in both PI and LGM simulations. 


\section{PMIP3 piControl sea surface temperature bias}

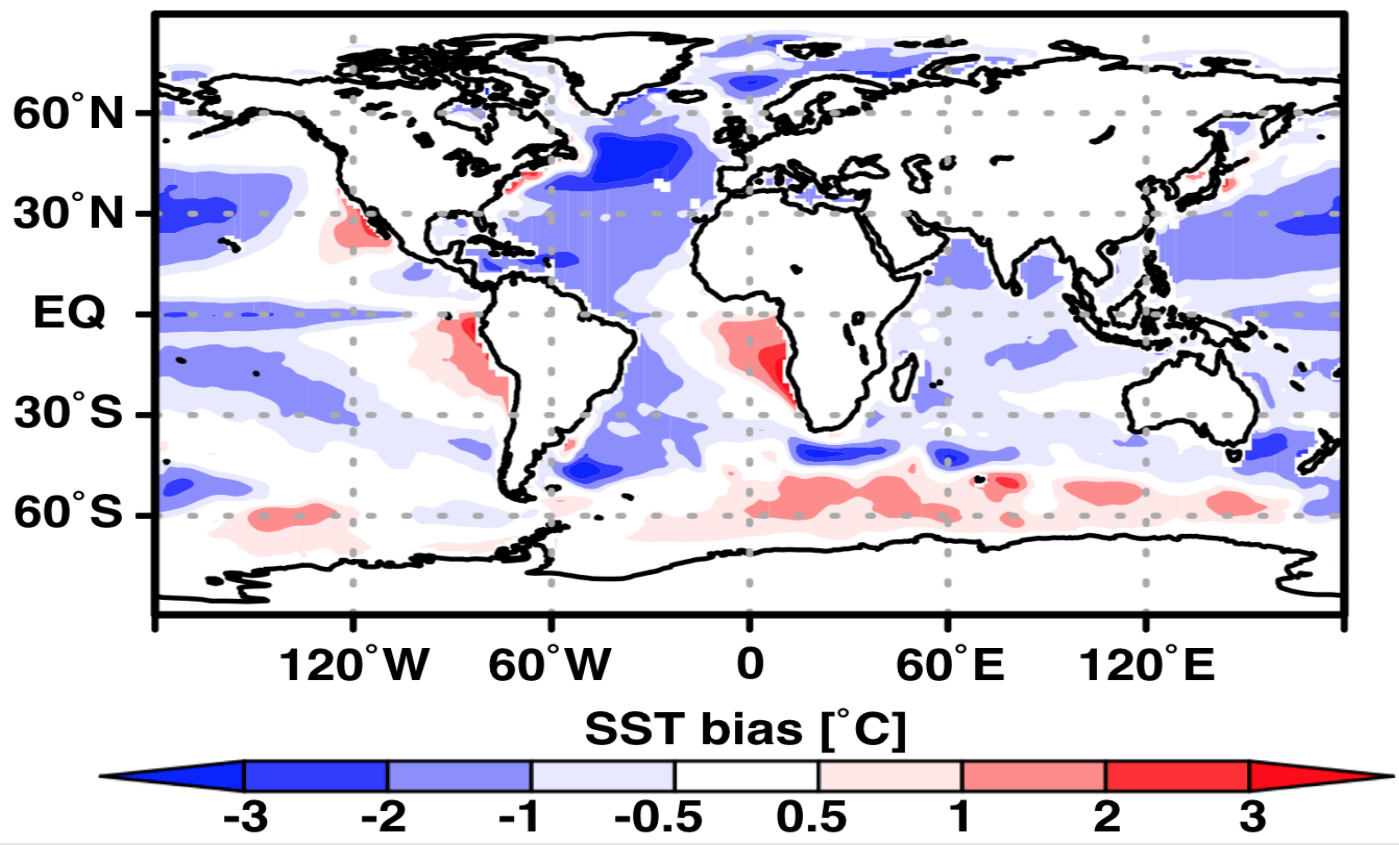

Figure 2: PMIP3 model mean annual sea-surface temperature bias in pre-industrial climate simulations compared with World Ocean Atlas 2013 (nodc.noaa.gov/OC5/woa13/ woa13data.html).

that modify the meridional density gradient under LGM conditions can be identified.

In the Southern Hemisphere, buoyancy loss through sea-ice export and brine release in the Southern Ocean associated with low $\mathrm{CO}_{2}$ concentrations are key for the formation of dense AABW (Klockmann et al. 2016). Models with a shallower LGM AMOC tend to have a very strong buoyancy loss over the Southern Ocean (Otto-Bliesner et al. 2007). An additional factor could be the duration of the spin-up: a sufficient integration time is required to account for the slow penetration and densification of the deep Atlantic by AABW (Marzocchi and Jansen 2017).

In the Northern Hemisphere, key processes are changes in the North Atlantic freshwater budget, sea-ice cover, and surface winds. Stronger LGM surface winds over the North Atlantic caused by the Laurentide ice sheet increase the density and formation of NADW and induce a strong and deep LGM AMOC (Muglia and Schmittner 2015; Sherriff-

Tadano et al. 2018). Extensive sea-ice cover or increased freshwater input in the NADW formation sites reduces the buoyancy loss and leads to less dense NADW and a weake and shallower AMOC (Oka et al. 2012; Weber et al. 2007). Depending on the model specifics, these mechanisms might compensate differently and lead to very different LGM states (Klockmann et al. 2018).

Few PMIP4 models simulate a substantial deepening of the LGM AMOC (Fig. 1). This improvement with respect to PMIP3 may imply that the models are making some progress in capturing the important processes and getting the balance right. Future analyses of the PMIP4 simulations will show whether this confidence is justified.

\section{Discussion}

The current ensemble of simulations across all PMIP phases contains 29 simulations from
26 different models. Only seven of these simulations capture the shallower LGM AMOC, and five were performed with models from the CCSM family (Fig.1). It is, therefore, reasonable to say that it remains a challenge for most AOGCMs to reproduce an LGM AMOC in agreement with reconstructions. Why is it so difficult?

There are several factors that affect the LGM AMOC, either because they affect the key mechanisms described above or through additional mechanisms. These factors are for example, uncertainties in the ice-sheet reconstructions, the magnitude and representation of glacial tidal mixing (Peltier and Vettoretti 2014), or assumptions of the AMOC being in a quasi-equilibrium state with $21 \mathrm{ka}$ climate forcing (Zhang et al. 2013). The PMIP4 protocol explicitly addressed the uncertainties in the ice-sheet reconstructions by offering a choice between three different reconstructions: ICE6G, GLAC1D, and the previous PMIP3 ice sheets (Kageyama et al. 2017 and references therein). Most PMIP4 simulations were run with the ICE6G ice sheets; only two models were used for multiple simulations with different ice sheets. In these two models, the different ice-sheet reconstructions make only a small difference for the simulated LGM AMOC, but this need not be the case for other models or other ice-sheet reconstructions.

Additional problems could arise from biases in the pre-industrial control simulations. Figure 2 shows sea-surface temperature (SST) biases in pre-industrial climate simulations from PMIP3 models. Large SST biases are evident over the Southern Ocean and northern North Atlantic, where AABW and NADW are formed, respectively. A recent study with an AOGCM showed, in fact, that an improvement in modern SST biases over the Southern Ocean could help to reproduce the shallower LGM AMOC by enhancing the formation of AABW (Sherriff-Tadano et al. submitted). NADW formation areas experience large changes in surface winds at the LGM; hence, biases in this region require additional attention as well.

In the future, sensitivity experiments such as parameter ensembles, or partially coupled experiments, may provide useful information regarding the role of uncertain climate parameters and model biases in LGM simulations. Increased direct modeling of carbon isotopes and relevant tracers will be key for model-data comparisons, and to better understand and constrain the LGM AMOC, including its strength.

\section{AFFILIATIONS}

'School of Earth and Environment, University of Leeds, UK

${ }^{2}$ Institute of Coastal Systems - Analysis and Modelling Helmholtz-Zentrum Hereon, Geesthacht, Germany

\section{CONTACT}

Sam Sherriff-Tadano: S.Sherriff-Tadano@leeds.ac.uk Marlene Klockmann: marlene.klockmann@hereon.de REFERENCES

Kageyama M et al. (2017) Geosci Model Dev 10: 4035-4055

Kageyama M et al. (2021) Clim Past 17: 1065-1089

Kim SJ (2004) Clim Dyn 22: 639-651

Klockmann M et al. (2016) Clim Past 12: 1829-1846 Klockmann M et al. (2018) J Clim 31: 7969-7984

Lynch-Stieglitz J (2017) Ann Rev Mar Sci 9: 83-104

Marzocchi A, Jansen MF (2017) Geophys Res Lett 44: 6286-6295

Muglia J, Schmittner A (2015) Geophys Res Lett 42 9862-9869

Oka A et al. (2012) Geophys Res Lett 39: L09709 Otto-Bliesner B et al. (2007) Geophys Res Lett 34: 6

Peltier R, Vettoretti G (2014) Geophys Res Lett 41 : 7306-7313

Sherriff-Tadano S et al. (2018) Clim Dyn 50: 2881-2903

Weber $S$ et al. (2007) Clim Past 3: 51-64

Zhang X et al. (2013) Clim Past 9: 2319-2333 